Editor's Note: These short reviews of a recent paper in the Journal, written exclusively by graduate students or postdoctoral fellows, are intended to mimic the journal clubs that exist in your own departments or institutions. For more information on the format and purpose of the Journal Club, please see http://www.jneurosci.org/misc/ifa_features.shtml.

\title{
Nonglobal Homeostatic Synaptic Plasticity?
}

\author{
Jonathan Ting, ${ }^{1}$ Alexandra P. Few, ${ }^{2}$ and Kenneth Custer ${ }^{1}$ \\ ${ }^{1}$ Graduate Program in Neurobiology and Behavior and 2Department of Pharmacology, University of Washington, Seattle, Washington 98195 \\ Review of Moulder et al. (http://www.jneurosci.org/cgi/content/full/26/24/6618)
}

\section{Introduction}

Recent work supports the idea that homeostatic synaptic plasticity mechanisms are invoked to ensure that neurons continually operate within a dynamic physiological range of activity (Burrone and Murthy, 2003; Turrigiano and Nelson, 2004). Such adaptive changes can act to normalize overall neuronal activity after synapse-specific Hebbian potentiation or depression, or after aberrant excitation such as seizures. The predominance of evidence supports postsynaptic mechanisms for homeostatic synaptic plasticity, although some presynaptic mechanisms have been reported (Burrone and Murthy, 2003; Turrigiano and Nelson, 2004). Previously, Moulder et al. (2004) reported presynaptic homeostatic plasticity in response to brief pathophysiological depolarization. Now, these authors in The Journal of Neuroscience (Moulder et al., 2006) demonstrate that a similar presynaptic homeostatic plasticity can be induced by chronic changes in physiological activity and occurs in an all-or-none manner at individual synapses.

Moulder et al. (2006) examined synaptic function after either chronic silencing of neuronal activity with TTX and glutamate receptor antagonists, or chronic stimulation of neuronal activity by elevating potassium levels. Using cultured hippocampal neurons, the authors first measured the percentage of excitatory

Received Aug. 30, 2006; revised Sept. 14, 2006; accepted Sept. 16, 2006. Correspondence should be addressed to Jonathan Ting, University of Washington School of Medicine, Department of Physiology and Biophysics, P.0. Box 357290, Seattle, WA 98195. E-mail: jtting@u.washington.edu. DOI:10.1523/JNEUROSCI.3781-06.2006

Copyright $\odot 2006$ Society for Neuroscience $\quad$ 0270-6474/06/2610937-02\$15.00/0 synapses (vGluT-1-positive puncta) that were active (FM1-43 $\mathrm{Fx}$ positive). Chronic blockade of neuronal activity increased the percentage of active terminals [Moulder et al. (2006), their Fig. 1 (http://www. jneurosci.org/cgi/content/full/26/24/ $6618 / \mathrm{F} 1)]$, whereas chronic depolarization decreased the percentage of active terminals [Moulder et al. (2006), their Fig. 3 (http://www.jneurosci.org/cgi/content/ full/26/24/6618/F3)]. In the latter case, the remaining functional terminals had a similar distribution of FM1-43 $3_{\mathrm{FX}}$ staining intensity, indicating that the effects of chronic depolarization are all-or-none. Depolarization-induced inactivation of synapses was inhibited by TTX [Moulder et al. (2006), their Fig. 3C (http://www. jneurosci.org/cgi/content/full/26/24/ $6618 / F 3)]$, and field stimulation was also sufficient to induce synapse inactivation [Moulder et al. (2006), their Fig. $6 B$ (http://www.jneurosci.org/cgi/content/ full/26/24/6618/F6)]. Thus, spiking was necessary and sufficient to induce this form of homeostatic plasticity. Moreover, the regulation of presynaptic function was bidirectional, in a homeostatic, but synapse-specific, all-or-none manner.

To shed light on the underlying mechanisms, Moulder et al. (2006) measured evoked EPSCs, the frequency of miniature EPSCs (mEPSCs), and the sucroseevoked readily releasable pool of vesicles (RRP). All three were reduced after chronic depolarization, consistent with a decrease in the number of functional release sites [Moulder et al. (2006), their Figs. 5 (http://www.jneurosci.org/ cgi/content/full/26/24/6618/F5) and 6 (http://www.jneurosci.org/cgi/content/
full/26/24/6618/F6)]. It would have been interesting to test whether the remaining terminals had normal release properties by measuring synaptic release probability, vesicular release probability, and shortterm plasticity. Furthermore, a decrease in the total number of synapses could also contribute to the decreased EPSC amplitude, because the authors did not report total synapse numbers.

There was no difference in mEPSC size or in currents evoked by exogenous application of glutamate between control and depolarized neurons [Moulder et al. (2006), their Figs. $4 E$ (http://www.jneurosci.org/ cgi/content/full/26/24/6618/F4) and $5 D$ (http://www.jneurosci.org/cgi/content/ full/26/24/6618/F5)], suggesting there is no postsynaptic component to the adaptation they observed. Thus, the mechanism of homeostatic adaptation induced by chronic mild depolarization appears to be exclusively presynaptic. In contrast, Gomperts et al. (2000) showed that chronic activity blockade by TTX (without glutamate receptor antagonists) decreased mEPSC amplitudes, suggesting that under slightly different conditions suppressing neuronal activity may activate postsynaptic plasticity mechanisms.

The decreased RRP size after chronic stimulation could be attributable either to a decrease in the number of vesicles docked at the presynaptic active zone or downregulation of the vesicle priming processes that render docked vesicles release competent. To distinguish between these possibilities, Moulder et al. (2006) measured the number of morphologically docked vesicles in electron micrographs from control and depolarized neurons 
[Moulder et al. (2006), their Fig. 7 (http:// www.jneurosci.org/cgi/content/full/26/ 24/6618/F7)]. They found no difference between treatments, thus implicating a deficit in vesicle priming. This was confirmed through the used of $\alpha$-latrotoxin ( $\alpha$-LTX), a toxin that bypasses some priming steps to induce docked vesicles to fuse. In the presence of $\alpha$-LTX, the frequency of mEPSCs in chronically depolarized neurons was normal, indicating that presynaptic homeostatic adaptation induced by chronic spiking involves regulation of vesicle priming.

Moulder et al. (2006) provide evidence that the percentage of functional excitatory presynaptic terminals can be bidirectionally regulated by chronic changes in spiking (Fig. 1). Interestingly, this result also implies that, under "normal" conditions, basal spiking activity sustains a population of functionally inactive terminals. By comparing sucrose-evoked release and $\alpha$-LTX-evoked release, Moulder et al. (2006) further clarify that physiological activity regulates the percentage of functional release sites by acting on vesicle priming. It is of particular interest that neurons lacking the presynaptic proteins Bassoon or Munc13-1 exhibit a similar priming deficit to that induced by chronic increases in spiking, perhaps suggesting that the function of these proteins is compromised as a consequence of altered spiking rate.

The work by Moulder et al. (2006) also raises the possibility that data obtained from studies of knock-out mice may in part reflect adaptive homeostatic changes. For example, deleting a gene that normally suppresses neuronal activity may ultimately lead to a homeostatic decrease in synaptic function. In light of this, it seems ever important to employ rescue strategies, such as those demonstrated for Munc13-1 (Rosenmund et al., 2002), to verify that acute reintroduction of the protein of interest restores normal neurotransmission.

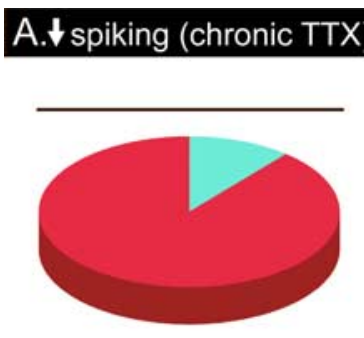

B. 'normal' spiking

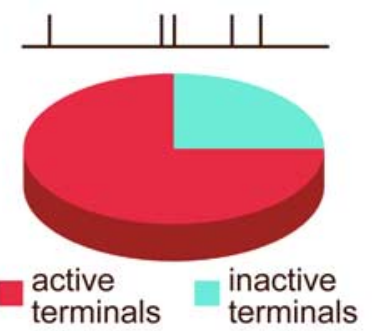

C. $\uparrow$ spiking (chronic $\mathbf{\uparrow K}^{+}$)

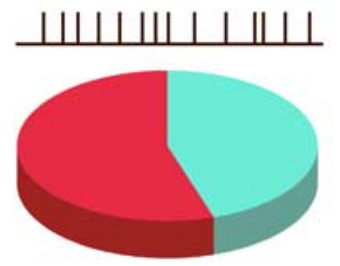

D. Presynaptic vesicle priming is regulated by changes in spiking rate

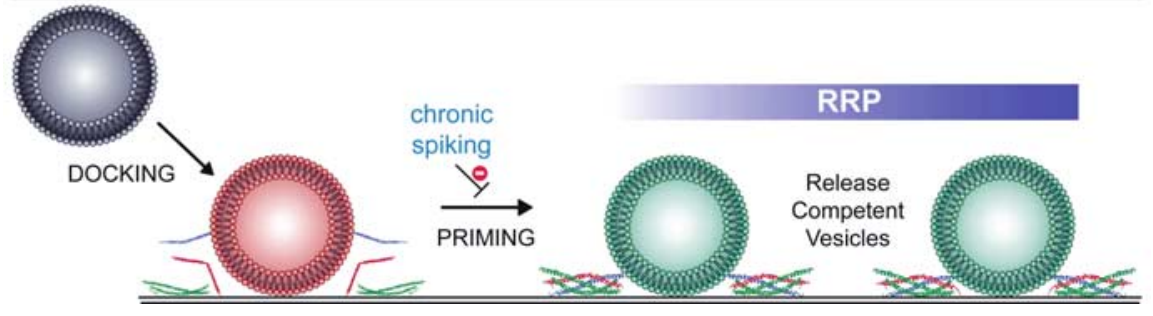

Figure 1. A-C, The percentage of functional presynaptic terminals is bidirectionally regulated by spiking rate. "Normal" spiking sustains a population of inactive terminals. The percentage of inactive terminals decreases in response to a chronic blockade of spiking, whereas a chronic increase in spiking increases the percentage of inactive terminals. $\boldsymbol{D}$, Spiking regulates presynaptic function by acting on synaptic vesicle priming. Vesicles normally undergo sequential docking and priming reactions before reaching a release competent state. The release competent vesicles collectively constitute the RRP. Chronic increases in spiking diminish the RRP by inhibiting vesicle priming.

Perhaps the most intriguing aspect of the work by Moulder et al. (2006) is that the adaptive mechanism under investigation is not global in expression (i.e., only a fraction of presynaptic terminals were affected by chronic manipulations in spiking, whereas the remaining fraction of terminals were resistant). This conflicts with the widely accepted notion that adaptive changes are the result of a cell-wide signal that impacts all synapses equally. In considering the overall impact on synaptic function, this nonglobal adaptive plasticity mechanism could serve to dampen runaway excitation. However, because not all synapses are affected equally, it seems unlikely such a mechanism can also preserve information about the relative strength of individual synapses. Additional work is necessary to clarify the roles of global versus nonglobal forms of homeostatic plasticity.

\section{References}

Burrone J, Murthy VN (2003) Synaptic gain control and homeostasis. Curr Opin Neurobiol 13:560-567.

Gomperts SN, Carroll R, Malenka RC, Nicoll RA (2000) Distinct roles for ionotropic and metabotropic glutmate receptors in the maturation of excitatory synapses. J Neurosci 20:2229-2237.

Moulder KL, Meeks JP, Shute AA, Hamilton CK, de Erausquin G, Mennerick S (2004) Plastic elimination of functional glutamate release sites by depolarization. Neuron 42:423-435.

Moulder KL, Jiang X, Taylor AA, Olney JW, Mennerick S (2006) Physiological activity depresses synaptic function through an effect on vesicle priming. J Neurosci 26:6618-6626.

Rosenmund C, Sigler A, Augustin I, Reim K, Brose N, Rhee JS (2002) Differential control of vesicle priming and short-term plasticity by Munc13 isoforms. Neuron 33:411-424.

Turrigiano GG, Nelson SB (2004) Homeostatic plasticity in the developing nervous system. Nat Rev Neurosci 5:97-107. 